\title{
Multipolar superconductivity in Luttinger semimetals
}

\author{
GiBaik Sim, ${ }^{1}$ Archana Mishra, ${ }^{1,2}$ Moon Jip Park, ${ }^{1}$ Yong Baek Kim, ${ }^{3,4}$ Gil Young Cho, ${ }^{5}$ and SungBin Lee $\odot^{1}$ \\ ${ }^{1}$ Department of Physics, Korea Advanced Institute of Science and Technology, Daejeon 305-701, Korea \\ ${ }^{2}$ International Research Centre MagTop, Institute of Physics, Polish Academy of Sciences, Aleja Lotników 32/46, PL-02668 Warsaw, Poland \\ ${ }^{3}$ Department of Physics, University of Toronto, Toronto, Ontario, Canada M5S $1 A 7$ \\ ${ }^{4}$ School of Physics, Korea Institute for Advanced Study, Seoul 02455, Korea \\ ${ }^{5}$ Department of Physics, POSTECH, Pohang, Gyeongbuk 790-784, Korea
}

(Received 21 January 2020; revised manuscript received 3 June 2020; accepted 5 June 2020;

published 30 June 2020)

\begin{abstract}
Topological superconductivity in multiband systems has received much attention due to a variety of possible exotic superconducting order parameters as well as nontrivial bulk and surface states. While the impact of coexisting magnetic order on superconductivity, such as ferromagnetic superconductors, has been studied for many years, the implication of coexisting multipolar order has not been explored much despite the possibility of multipolar hidden order in a number of $f$-electron materials. In this work, we investigate topological properties of multipolar superconductors that may arise when quadrupolar local moments are coupled to conduction electrons in the multiband Luttinger semimetal. We show that the multipolar ordering of local moments leads to various multipolar superconductors with distinct topological properties. We apply these results to the quadrupolar Kondo semimetal system, PrBi, by deriving the microscopic multipolar Kondo model and examining the possible superconducting order parameters.
\end{abstract}

DOI: 10.1103/PhysRevResearch.2.023416

\section{INTRODUCTION}

One of the foremost themes in contemporary condensedmatter physics is the realization of topological superconductivity, where Bogoliubov-de Gennes (BdG) quasiparticles are characterized by nontrivial topology [1-4]. Among the numerous proposals to realize the topological superconductors [5-11], a prominent route is to utilize multiband or multiorbital superconductivity [12-35], where the Cooper pairs possess nonzero angular momentum through the interband pairing channels. A representative example is the superconductivity in pseudospin $j=3 / 2$ Luttinger semimetals [36,37] with low-energy excitations described by quadratic band touching.

The multiband nature of the Luttinger semimetals has motivated intensive research on the possible unconventional superconductors supporting the Cooper pairs with higher pseudospin angular momentum $j$ [12-24]. In particular, it has been shown that the electron-electron interaction favors the $d$-wave pairing channels in the $j=2$ manifold over the $s$ wave in the $j=0$ state [19]. Such unconventional superconductors possess a number of striking features, including the emergent topological boundary states and the Bogoliubov Fermi surfaces with nontrivial Chern numbers [13-15,38]. All these interesting properties arise uniquely in multiband

Published by the American Physical Society under the terms of the Creative Commons Attribution 4.0 International license. Further distribution of this work must maintain attribution to the author(s) and the published article's title, journal citation, and DOI. systems and result from the interplay between spin-orbit coupling and interband pairing channels [13,22]. Among various candidate materials, half-Heusler compounds such as YPtBi, $\mathrm{LuPdBi}$, and $\mathrm{LaBiP}$ belong to such multiband superconductors [39-41]. These half-Heusler compounds have negligible anisotropies of the Fermi surface near the quadratic bandtouching point $[42,43]$. Therefore, the Luttinger model with $\mathrm{SO}(3)$ and cubic symmetries have been employed to explain the superconductivity in these materials $[12,17,19,23]$.

On the other hand, unlike the half-Heuslers addressed above, other series of half-Heuslers like TbPdBi and HoPdBi exhibit unconventional superconductivity coexisting with magnetic ordering from the rare-earth ions $\mathrm{Tb}$ and Ho [44,45]. These materials are extremely interesting platforms for the study of the interplay between the magnetic degrees of freedom and unconventional superconductivity in multiorbital systems. Furthermore, the pyrochlore oxide $\mathrm{Cd}_{2} \mathrm{Re}_{2} \mathrm{O}_{7}$ and Pr-based intermetallic compounds $\operatorname{Pr}(T)_{2} X_{20}(T=\mathrm{Ti}, \mathrm{V}, \mathrm{Rh}$, Ir and $X=\mathrm{Al}, \mathrm{Zn}$ ) were recently found to show the coexistence of multipolar order and superconductivity [46-57]. Another semimetallic system, $\mathrm{PrBi}$, is known to have both the quadrupolar degrees of freedom coming from Pr ions and the $j=3 / 2$ Luttinger semimetal. Recent experiments on this material have confirmed the existence of ferroquadrupolar order originating from the localized moments of Pr ions, which may indicate the importance of the quadrupolar Kondo effect [58]. Such a situation is analogous to ferromagnetic superconductors, where the presence of magnetism can significantly alter the nature of the superconducting state. Hence, it is conceivable that the presence of multipolar order could change the nature of the resulting multipolar superconductors in some fundamental ways. 
In this paper, motivated by the intertwined physics of multipolar order and superconductivity, we discuss how their coexistence can give rise to multipolar superconductivity with unique topological properties. In particular, we consider the PrBi system as a concrete example and derive the microscopic quadrupolar Kondo model, where the non-Kramers doublet of the localized Pr moments and the Bi itinerant electrons described by the Luttinger model interact with each other. In the absence of quadrupolar order, we first discuss the superconducting phases within the cubic-symmetric Luttinger model. We find that time-reversal symmetry-breaking $d$-wave superconductors occur in the weak-coupling limit, while the time-reversal symmetry is restored in the strong-coupling limit. In the presence of quadrupolar order, however, we find that the superconducting instabilities are significantly altered in a way that the quadrupolar order induces Fermi-surface distortion and stabilizes the multipolar superconductivity with mixtures of distinct $d$-wave pairing order parameters. Moreover, we find that these superconducting phases harbor topologically nontrivial gapless nodal-line or nodal-surface excitations, the nature of which sensitively depends on the quadrupolar order. Thus, one could change the topological properties of the multipolar superconductors by controlling the coexisting multipolar order. This would be a good example of topological phases that could be controlled by multipolar degrees of freedom. Based on our theory, we also propose various experiments that can probe the topological nature of the Bogoliubov quasiparticles in multipolar superconductors, anticipating potential applications to PrBi materials with doping and external pressure.

\section{LUTTINGER MODEL AND ELECTRON INTERACTION}

We start by describing the kinetics of the itinerant electrons with the Luttinger-semimetal Hamiltonian,

$$
H_{0}(\boldsymbol{k})=c_{0} k^{2}+\sum_{i=1}^{5} c_{i} d_{i}(\boldsymbol{k}) \gamma_{i}-\mu,
$$

in the four-component spinor basis defined as $\psi \equiv\left(\psi_{3 / 2}\right.$, $\left.\psi_{1 / 2}, \psi_{-1 / 2}, \psi_{-3 / 2}\right)$ and with the five $4 \times 4$ anticommuting Dirac matrices $\gamma_{i}$ [59]. Here, $\mu$ is the chemical potential, $d_{i}(\boldsymbol{k})$ represents the five real $l=2$ spherical harmonics, with $d_{1}=\sqrt{3}\left(k_{x}^{2}-k_{y}^{2}\right) / 2, \quad d_{2}(\boldsymbol{k})=\left(3 k_{z}^{2}-\boldsymbol{k}^{2}\right) / 2, \quad d_{3}(\boldsymbol{k})=$ $\sqrt{3} k_{y} k_{z}, d_{4}(\boldsymbol{k})=\sqrt{3} k_{z} k_{x}$, and $d_{5}(\boldsymbol{k})=\sqrt{3} k_{x} k_{y}$. The Dirac matrices $\gamma_{i}$ are explicitly given as $\gamma_{1}=\sigma^{x} \otimes \mathbb{I}, \gamma_{2}=\sigma^{z} \otimes$ $\sigma^{z}, \gamma_{3}=\sigma^{z} \otimes \sigma^{y}, \gamma_{4}=\sigma^{z} \otimes \sigma^{x}$, and $\gamma_{5}=\sigma^{y} \otimes \mathbb{I}$, where $\sigma^{\alpha}$ are the Pauli matrices and $\mathbb{I}$ is the $2 \times 2$ identity matrix. It is worth noting that Eq. (1) is a complete representation of kinetics in the Luttinger semimetal when both inversion symmetry and time-reversal symmetry are present. In Eq. (1), $c_{0}$ quantifies the particle-hole asymmetry of the model, whereas $c_{i}$ quantify the kinetic terms proportional to each of the $d$ wave harmonics. When all $c_{i}$ are the same, the model in Eq. (1) becomes fully spherically symmetric, retaining $\mathrm{SO}(3)$ symmetry. In the case of cubic symmetry, however, we have $c_{1,2} \neq c_{3,4,5}[23]$.

We now discuss the superconductivity emerging from this multiband Luttinger semimetal when the electron-electron interactions are present [19],

$$
H_{I}=g_{0}\left(\psi^{\dagger} \psi\right)^{2}+\sum_{a} g_{a}\left(\psi^{\dagger} \gamma_{a} \psi\right)^{2} .
$$

Using the Fierz identity, $H_{I}$ can be exactly rewritten in terms of the $s$-wave and $d$-wave pairing channels, $H_{I}=H_{s}+$ $\sum_{a} H_{d_{a}}$, with

$$
\begin{aligned}
H_{s} & =g_{s}\left(\psi^{\dagger} \gamma_{45} \psi^{*}\right)\left(\psi^{T} \gamma_{45} \psi\right), \\
H_{d_{a}} & =g_{d_{a}}\left(\psi^{\dagger} \gamma_{a} \gamma_{45} \psi^{*}\right)\left(\psi^{T} \gamma_{45} \gamma_{a} \psi\right),
\end{aligned}
$$

where $g_{s}=\frac{1}{4}\left(g_{0}+\sum_{a} g_{a}\right), \quad g_{d_{a}}=\frac{1}{4}\left(g_{0}+g_{a}-\sum_{b \neq a} g_{b}\right)$, and $\gamma_{45}=i \gamma_{4} \gamma_{5}$. It is remarkable that the repulsive electron interaction with coefficients $g_{a}>0$ can naturally induce the $d$-wave pairing instabilities [19]. For simplicity, we set coefficients $g_{a}=g_{1}$, and hence, $g_{s}=\frac{1}{4}\left(g_{0}+5 g_{1}\right)$, and $g_{d_{a}}=\frac{1}{4}\left(g_{0}-3 g_{1}\right)$. In this work, we assume that the $d$-wave pairing channel is attractive such that $g_{d_{a}}=-g$ and neglect $H_{s}$. Within the standard mean-field decomposition, $H_{I}$ can be rewritten as follows up to the constant terms:

$$
H_{I}=-g \sum_{a}\left\{\left(\psi^{\dagger} \gamma_{a} \gamma_{45} \psi^{*}\right) \Delta_{a}+\left(\psi^{T} \gamma_{45} \gamma_{a} \psi\right) \Delta_{a}^{*}\right\},
$$

where the superconducting order parameters are explicitly given as

$$
\Delta_{a}=\left\langle\psi_{-k}^{T} \gamma_{45} \gamma_{a} \psi_{k}\right\rangle .
$$

The order parameter $\Delta_{a}$, with $a \in(1,2, \ldots, 5)$, represents the $d$-wave quintet pairings $(j=2)$. In particular, $\Delta_{e_{g}} \equiv\left(\Delta_{1}, \Delta_{2}\right)$ represents the two $d$-wave pairings, $\left(d_{x^{2}-y^{2}}, d_{3 z^{2}-r^{2}}\right)$ with $e_{g}$ symmetry, and $\Delta_{t_{2 g}} \equiv\left(\Delta_{3}, \Delta_{4}, \Delta_{5}\right)$ represents the three $d$ wave pairings, $\left(d_{y z}, d_{z x}, d_{x y}\right)$ with $t_{2 g}$ symmetry. Throughout our study, we consider the specific parameter set, which is relevant to $\mathrm{PrBi}$, in Eq. (1) and analyze the properties of superconducting states; $c_{0}=-6(a / \pi)^{2} \mathrm{eV}, c_{e_{8}} \equiv c_{1}=c_{2}=$ $-2(a / \pi)^{2} \mathrm{eV}$, and $c_{t_{28}} \equiv c_{3}=c_{4}=c_{5}=-1(a / \pi)^{2} \mathrm{eV}$, with the lattice constant $a$ and the chemical potential $\mu=-0.6 \mathrm{eV}$, for the cubic-symmetric case $[60,61]$. Here and below, we consider the case where there are two distinct doubly degenerate Fermi surfaces for $\mu<0$ (normal band structure). Although we focus on the specific parameter set, we emphasize that a similar argument holds for different cases, and the emergence of complex superconducting states due to intertwined multipolar order is a generic feature.

We first briefly discuss the superconducting phases in the absence of coexisting quadrupolar order, with the cubicsymmetric Luttinger model where the coefficients in Eq. (1) are of the form $\left|c_{e_{g}}\right|>\left|c_{t_{28}}\right|$. In general, the free energy for the $\Delta_{e_{g}}$ pairing state is given as $F_{e_{g}}=r_{e_{g}} \Delta_{e_{g}} \cdot \Delta_{e_{g}}^{*}+q_{1}\left(\Delta_{e_{g}}\right.$. $\left.\Delta_{e_{g}}^{*}\right)^{2}+q_{2}\left(\Delta_{1} \Delta_{2}^{*}-\Delta_{2} \Delta_{1}^{*}\right)^{2}+s_{1}\left(\Delta_{e_{g}} \cdot \Delta_{e_{g}}^{*}\right)^{3}+s_{2}\left(\Delta_{e_{g}} \cdot \Delta_{e_{g}}^{*}\right)$ $\left|\Delta_{e_{g}} \cdot \Delta_{e_{g}}\right|^{2}+s_{3}\left|\Delta_{2}\right|^{2}\left|3 \Delta_{1}^{2}-\Delta_{2}^{2}\right|^{2}$, while the free energy for the $\Delta_{t_{2 g}}$ pairing state is given as $F_{t_{2 g}}=r_{t_{2 g}} \Delta_{t_{2 g}} \cdot \Delta_{t_{2 g}}^{*}+$ $q_{1}^{\prime}\left(\Delta_{t_{2 g}} \cdot \Delta_{t_{2 g}}^{*}\right)^{2}+q_{2}^{\prime}\left|\Delta_{t_{2 g}} \cdot \Delta_{t_{2 g}}\right|^{2}+q_{3}^{\prime}\left(\left|\Delta_{3}\right|^{2}\left|\Delta_{4}\right|^{2}+\left|\Delta_{4}\right|^{2}\left|\Delta_{5}\right|^{2}\right.$ $\left.+\left|\Delta_{5}\right|^{2}\left|\Delta_{3}\right|^{2}\right)$ [62]. Within one-loop calculation, the instability towards the $\Delta_{e_{p}}$ pairing is shown to be stronger than the $\Delta_{t_{2 g}}$ pairing with $\left|c_{e_{g}}\right|>\left|c_{t_{2 g}}\right|$, i.e., $r_{e_{g}}<r_{t_{2 g}}$ (see Appendix A 1 for details). For the $\Delta_{e_{g}}$ pairing, there are three possible superconducting states with the order parameters $\Delta_{e_{g}}=(1,0),(0,1)$, and $(1, i)$. By comparing the 


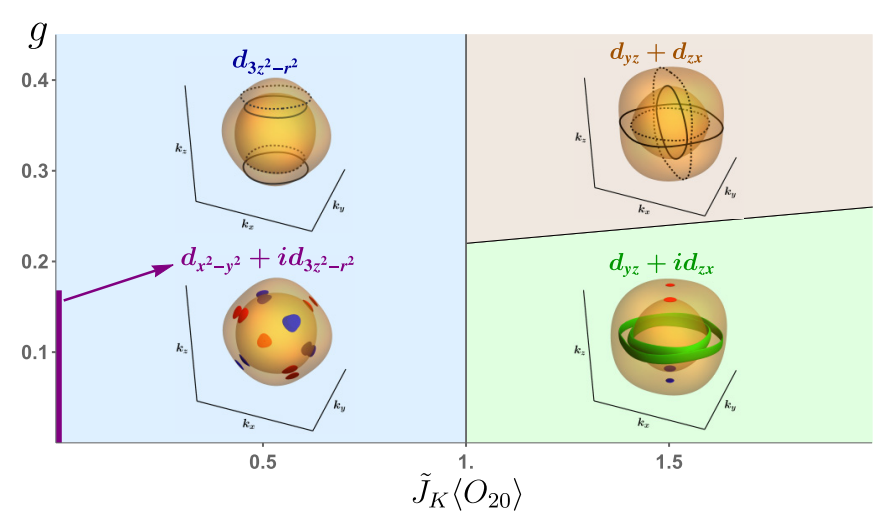

FIG. 1. Multipolar superconducting phases as functions of $\tilde{J}_{K}\left\langle O_{20}\right\rangle\left[(a / \pi)^{2} \mathrm{eV}\right]$ and the interaction strength $g\left(a^{3} \mathrm{eV}\right)$ with $c_{0}=-6(a / \pi)^{2} \mathrm{eV}, c_{e_{g}}=c_{1}=c_{2}=-2(a / \pi)^{2} \mathrm{eV}, c_{\eta}=c_{3}=c_{4}=$ $\left(-1-\tilde{J}_{K}\left\langle O_{20}\right\rangle\right)(a / \pi)^{2} \mathrm{eV}, c_{5}=-1(a / \pi)^{2} \mathrm{eV}$, and $\mu=-0.6 \mathrm{eV}$ based on Eqs. (1) and (7). We find four distinct superconducting states: the time-reversal symmetry-breaking state with $d_{x^{2}-y^{2}}+$ $i d_{3 z^{2}-r^{2}}$ pairing (purple line) and $d_{y z}+i d_{z x}$ pairing (green) and the time-reversal symmetric state with $d_{3 z^{2}-r^{2}}$ pairing (blue) and $d_{y z}+$ $d_{z x}$ pairing (brown). Four insets show the gap structure of these states. Note that the $\left\langle O_{20}\right\rangle=0$ thick vertical line corresponds to the case with the cubic symmetry in the absence of quadrupolar order. Here, each semitransparent yellow surface represents the normal-state Fermi surface. For a multipolar superconductor with time-reversal symmetry breaking, the Bogoliubov Fermi surfaces exist. Red, green, and blue Bogoliubov Fermi surfaces indicate distinct Chern numbers 2,0 , and -2 , respectively. For the multipolar superconductor with time-reversal symmetry, each solid (dashed) ring indicates the nodal ring, which is protected by the nontrivial winding number $2(-2)$. See the text for more details.

mean-field energy at zero temperature, for the weak-coupling limit, i.e., small-g limit, we find that the time-reversal symmetry-breaking superconducting phase is chosen, which is described by $d_{x^{2}-y^{2}}+i d_{3 z^{2}-r^{2}}$ pairing or the order parameter $\Delta_{e_{g}}=(1, i)$ (see Appendix B for details). In this phase, the Bogoliubov quasiparticles form 16 distinct pockets, as shown in the bottom left inset of Fig. 1. Furthermore, we find that each pocket colored red (blue) is characterized by the nontrivial Chern number $2(-2)$, classified by the $2 \mathbb{Z}$ Chern number corresponding to class $\mathrm{D}$ in the Altland-Zirnbauer classification with additional inversion symmetry [63].

With increasing interaction strength $g$, we observe the superconducting phase transition occurs from the $d_{x^{2}-y^{2}}+$ $i d_{3 z^{2}-r^{2}}$ pairing state to the time-reversal-symmetric $d_{3 z^{2}-r^{2}}$ pairing state. This phase transition can be understood as the effect of band flattening near the quadratic band-touching point. More precisely, the electron interaction starts to dominate over the kinetic energy at large $g$, and the system behaves similarly to the case with small $\mu$ due to the band flattening near $\boldsymbol{k}=0$, which favors the $d_{3 z^{2}-r^{2}}$ pairing state [19]. In Fig. 1, the purple vertical thick line at the bottom left corresponds to $d_{x^{2}-y^{2}}+i d_{3 z^{2}-r^{2}}$ pairing, and there is a phase transition to $d_{3 z^{2}-r^{2}}$ beyond that, as the interaction strength $g$ increases, in the case with the cubic symmetry. The BdG energy spectrum of this phase possesses gapless nodal rings, as shown in the top left inset of Fig. 1. In this time-reversal-symmetric superconductor, the solid (dashed) nodal line is protected by the nontrivial winding number $2(-2)$, which belongs to the $2 \mathbb{Z}$ classification of the DIII class [63].

\section{MULTIPOLAR KONDO COUPLING}

When multipolar degrees of freedom are present in the system, one should consider an effective Kondo coupling between the localized multipolar moments and itinerant electrons. In this section, we consider the microscopic model focusing on PrBi and derive the multipolar Kondo coupling between the $e_{g}$-type quadrupolar moments in $\operatorname{Pr}^{3+}$ and the strongly spin-orbit coupled electrons of $\mathrm{Bi} 6 p$ orbitals. The $e_{g^{-}}$ type quadrupolar degrees of freedom in the cubic-symmetric model are represented in terms of the Stevens operators $O_{22}=$ $\frac{\sqrt{3}}{2}\left(J_{x}^{2}-J_{y}^{2}\right)$ and $O_{20}=\frac{1}{2}\left(3 J_{z}^{2}-J^{2}\right)$, with $J_{\mu}$ being the $\mu$ th component of total angular momentum [64,65]. Regarding $\mathrm{PrBi}$, we consider the interpenetrating fcc lattice system, where the quadrupolar degrees of freedom $\mathrm{O}_{22}$ and $\mathrm{O}_{20}$ of the localized electrons reside in one fcc lattice and the itinerant electrons with $p$ orbitals reside in another fcc lattice (for details, see Fig. 8). Then, one can write down the effective Kondo coupling between the quadrupolar order parameters $\mathrm{O}_{22}$ and $\mathrm{O}_{20}$ and the itinerant $p$ electrons as follows:

$$
H_{K}=J_{K} \sum_{\langle i, j\rangle} \sum_{a, \alpha}\left(O_{22} \Gamma_{1, i j}^{a} c_{i a \alpha}^{\dagger} c_{j a \alpha}+O_{20} \Gamma_{2, i j}^{a} c_{i a \alpha}^{\dagger} c_{j a \alpha}\right) .
$$

Here, $c_{i a \alpha}^{\dagger}$ and $c_{i a \alpha}$ are the electron creation and annihilation operators at site $i$ with orbital $a \in(x, y, z)$ and spin $\alpha \in$ $(\uparrow, \downarrow) . \Gamma_{1, i j}^{a}$ and $\Gamma_{2, i j}^{a}$ are site- and orbital-dependent form factors for the Kondo coupling with quadrupoles $\mathrm{O}_{22}$ and $\mathrm{O}_{20}$, respectively (see Appendix C for details). We note that the quadrupolar degrees of freedom, which are time reversal symmetric, can couple to only the spin-independent electron hoppings with the form factors that transform exactly the same as $O_{22}$ and $O_{20}$. Now we take into account the $j=3 / 2$ basis in the presence of the spin-orbit coupling of $p$ electrons and project Eq. (6) onto the $j=3 / 2$ basis with the projection operator $P_{j=3 / 2}$ [66]. Then one gets the following Kondo coupling:

$$
\begin{aligned}
\tilde{H}_{K}(\boldsymbol{k})= & P_{j=3 / 2} H_{K}(\boldsymbol{k}) P_{j=3 / 2} \\
= & \tilde{J}_{K}\left[\left(\sqrt{3} O_{20}+O_{22}\right) d_{3}(\boldsymbol{k}) \gamma_{3}\right. \\
& \left.+\left(\sqrt{3} O_{20}-O_{22}\right) d_{4}(\boldsymbol{k}) \gamma_{4}-2 O_{22} d_{5}(\boldsymbol{k}) \gamma_{5}\right]
\end{aligned}
$$

in the four-component spinor basis $\psi$ with $\tilde{J}_{K} \equiv J_{K}\left(\frac{a}{\pi}\right)^{2}$. In Eq. (7), one can clearly see that $O_{20}$-type ferroquadrupolar ordering breaks the threefold rotation symmetry, while $\mathrm{O}_{22}$-type ferroquadrupolar ordering breaks both the threefold and fourfold rotation symmetries. Recent experiment on the $\mathrm{PrBi}$ compound confirmed $\mathrm{O}_{20}$-type ferroquadrupolar order $\left\langle O_{20}\right\rangle \neq 0$, which has also been discussed within the Landau theory analysis on symmetry grounds $[58,67,68]$. Thus, we focus on the case when $O_{20}$-type ferroquadrupolar order is present, $\left\langle O_{20}\right\rangle \neq 0$ and $\left\langle O_{22}\right\rangle=0$. 


\section{FERROQUADRUPOLAR ORDER AND SUPERCONDUCTIVITY}

When $\left\langle O_{20}\right\rangle \neq 0$, the symmetry of the system is lowered to $D_{4 h}$ from the $O_{h}$ group $[69,70]$. One can easily see from both Eqs. (1) and (7) that ferroquadrupolar order gives rise to anisotropies in coefficients, $c_{3}=c_{4} \neq c_{5}$, and results in Fermi-surface distortion. In this case, the coefficient $c_{\eta} \equiv$ $c_{3}=c_{4}$ is renormalized in Eq. (1), and the spontaneous Fermisurface distortion occurs via the effective Kondo coupling shown in Eq. (7). In particular, when the quadrupolar order induces the Fermi-surface distortion, we find that the properties of the $d$-wave superconductivity are dramatically changed. In Fig. 1, we plot the phase diagram within the mean-field approximation as a function of $\tilde{J}_{K}\left\langle O_{20}\right\rangle$ and the interaction strength $g$ at zero temperature. With the onset of $O_{20}$-type ferroquadrupolar order, the instability towards the $\Delta_{2}$ pairing is shown to be stronger than the $\Delta_{1}$ pairing, which is consistent with the result of the one-loop calculation (see Appendix A 1 for details). Thus, the system prefers the $d_{3 z^{2}-r^{2}}$ pairing with $\Delta_{e_{g}}=(0,1)$ in both weak- and strong-coupling limits. With a further increase of $\tilde{J}_{K}\left\langle O_{20}\right\rangle$, however, the instability towards the $\Delta_{\eta} \equiv\left(\Delta_{3}, \Delta_{4}\right)$ pairing becomes stronger than the $\Delta_{2}$ pairing. In general, the free energy for the $\Delta_{\eta}$ pairing state is represented as $F_{\eta}=r_{\eta} \Delta_{\eta} \cdot \Delta_{\eta}^{*}+q_{1}\left(\Delta_{\eta} \cdot \Delta_{\eta}^{*}\right)^{2}+$ $q_{2}\left|\Delta_{\eta} \cdot \Delta_{\eta}\right|^{2}+q_{3}\left(\left|\Delta_{3}\right|^{4}+\left|\Delta_{4}\right|^{4}\right)$ [71]. Once the instability of the $\Delta_{\eta}$ pairing gets stronger than the $\Delta_{2}$ pairing, the phase transition to the $\Delta_{\eta}$ pairing occurs (see Appendix A 1 for details). For the weak-coupling limit, the system develops time-reversal symmetry- breaking superconductivity with the $d_{y z}+i d_{z x}$ pairing and the order parameters $\Delta_{\eta}=(1, i)$ (see Appendix B for details). This result is distinct from the cubic case, where the $d_{x^{2}-y^{2}}+i d_{3 z^{2}-r^{2}}$ pairing with $\Delta_{e_{g}}=(1, i)$ is chosen. As shown in the bottom right inset of Fig. 1, the Bogoliubov quasiparticles form four Fermi surfaces along the $k_{z}$ axis with Chern numbers \pm 2 and two Fermi surfaces located at $k_{z}=0$ with the Chern number 0 . With increasing $g$, the phase transition occurs, favoring distinct superconducting phase with the $d_{y z}+d_{z x}$ pairing described by the order parameter $\Delta_{\eta}=(1,1)$. In this case, the time-reversal symmetry is recovered, and the Bogoliubov quasiparticles form four nodal rings with the winding numbers \pm 2 , as shown in the top right inset of Fig. 1.

\section{DISCUSSION}

We have studied exotic multipolar superconductors and their topological properties, which arise from the intertwined multipolar order and electron correlations in the Luttinger semimetal. Based on the derivation of microscopic modeling of Kondo coupling between quadrupolar moments and conduction electrons, we found that the onset of ferroquadrupolar order affects the Fermi-surface distortion, and thereby multipolar superconductors occur in preferred $d$-wave superconducting order parameters. We emphasize that such phenomena are quite unique in the interacting Luttinger semimetals with relatively small carrier densities, where the effective Kondo coupling with the quadrupolar degrees of freedom can sensitively control the nature of the superconducting order parameters and the associated topological properties.
Recent experiments on the semimetallic compound PrBi have confirmed the existence of $O_{20}$-type ferroquadrupolar order below the transition temperature $T_{Q}=0.08 \mathrm{~K}$ [58]. In this material, the localized moments of $\mathrm{Pr}^{3+}$ ions form a $\Gamma_{3}$ non-Kramers doublet via strong spin-orbit coupling, which allows only higher multipolar moments but no dipole moment. However, the itinerant electrons of $\mathrm{Bi} 6 p$ orbitals form a strongly correlated Luttinger semimetal with small carrier density $[58,60]$. Since the system has a tiny carrier density, one may expect to control electron correlation via doping and external pressure, resulting in superconductivity driven by the interplay between the quadrupolar Kondo effect and the electron interaction. In such cases, as shown in Fig. 1, the multipolar superconductivity with distinct $d$-wave pairing order parameters is stabilized, and depending on the presence and absence of ferroquadrupolar order, the topological nature of the Bogoliubov quasiparticles may be sensitively changed. This can be verified by probing surface states such as Fermi arcs or drumheadlike surface states [21]. For time-reversal symmetry-breaking phases, the gapless quasiparticle excitation near the Bogoliubov Fermi surface would result in a finite residual specific heat and thermal conductivity at zero temperature resembling that of a normal metal [72-74]. Moreover, it was recently shown that the theoretical specific-heat calculation agrees well with the experiment on $\mathrm{FeSe}_{1-x} \mathrm{~S}_{x}$, which implies the presence of the Bogoliubov Fermi surface near the nematic quantum critical point [72]. Further experimental signatures to detect the existence of the Bogoliubov Fermi surface are summarized in Ref. [73].

With growing interest in multipolar order, often termed "hidden order," it is now known that there exist many systems where both multipolar order and superconductivity may coexist. For instance, beyond the quadrupolar Kondo semimetal $\mathrm{PrBi}$, materials like rare-earth half-Heusler compounds, $\mathrm{Pr}$ based cage compounds $\operatorname{Pr}(\mathrm{Ti}, \mathrm{V}, \mathrm{Ir})_{2}(\mathrm{Al}, \mathrm{Zn})_{20}$, and lacunar spinel compounds $\mathrm{Ga}(\mathrm{Ta}, \mathrm{Nb})_{4}(\mathrm{~S}, \mathrm{Se})_{8}$ contain spin-orbit entangled pseudospin degrees of freedom and sometimes exhibit (anti) ferroquadrupolar order in addition to superconductivity. In such cases, the multipolar Kondo coupling and strongly interacting multiorbital electrons play an important role in determining the characteristics of superconductivity. Our results can be used to understand how these two phenomena can be intertwined with each other and how the topological properties of multipolar superconductors could be controlled via the multipolar order. Our work provides an important platform for the discovery of multipolar topological superconductors that can be controlled by electron correlation or quadrupolar ordering.

\section{ACKNOWLEDGMENTS}

Y.B.K. is supported by the NSERC of Canada, Canadian Institute for Advanced Research, and Center for Quantum Materials at the University of Toronto. G.Y.C. is supported by the BK21 plus program, POSTECH. A.M. is supported by BK21 plus. G.B.S., M.J.P., and S.B.L. are supported by the KAIST startup, BK21, and a National Research Foundation grant (Grant No. NRF-2017R1A2B4008097). 


\section{APPENDIX A: GINZBURG-LANDAU FREE ENERGY AND ONE-LOOP EXPANSION}

\section{Quadratic coefficients}

In this section, we compute the coefficient of the quadratic term $r_{a}$ in the Ginzburg-Landau free energy $F\left(\Delta_{a}\right)$ to compare the strength of instabilities towards $\Delta_{a}$ pairing. We first introduce the free-electron propagator

$$
\begin{aligned}
G(K) & =\left[i k_{0}+c_{0} k^{2}+\sum_{i} c_{i} d_{i}(\boldsymbol{k}) \gamma_{i}-\mu\right]^{-1} \\
& =\frac{-i k_{0}-c_{0} k^{2}+\sum_{i} c_{i} d_{i}(\boldsymbol{k}) \gamma_{i}+\mu}{\sum_{i}\left(c_{i} d_{i}(\boldsymbol{k})\right)^{2}-\left(c_{0} k^{2}+i k_{0}-\mu\right)^{2}}
\end{aligned}
$$

Here $K \equiv\left(k_{0}, \boldsymbol{k}\right)$, and $k_{0}=2 \pi(n+1 / 2) T$ denotes the Matsubara frequency. Then, the free energy is written as

$$
F(\vec{\Delta})=\frac{1}{g}|\vec{\Delta}|^{2}+T \sum_{m, n} \int_{k}^{\Lambda} \frac{1}{m} \operatorname{tr}\left[-G(K) \hat{\Delta} G(-K)^{T} \hat{\Delta}^{\dagger}\right]^{m},
$$

where $\hat{\Delta}=\sum_{a} \gamma_{a} \gamma_{45} \Delta_{a}$.

Let $F_{2}\left(\Delta_{a}\right)$ be the contribution to the free energy that contains the second power of $\Delta_{a}$. We have

$$
F_{2}\left(\Delta_{a}\right)=\frac{1}{g}\left|\Delta_{a}\right|^{2}-\frac{1}{2} L_{a} \Delta_{a}^{*} \Delta_{a},
$$

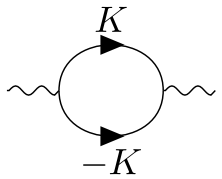

FIG. 2. Diagrammatic representation of $L_{a}$. Each solid arrow refers to the free-electron propagator $G(K)$, with $K=\left(k_{0}, \boldsymbol{k}\right)$, while each wiggly line indicates the insertion of $\Delta_{a}$ with vertex $\gamma_{a}$.

with

$$
L_{a}=T \sum_{k_{0}} \int_{k}^{\Lambda} \operatorname{tr}\left[G(K) \gamma_{a} G(-K) \gamma_{a}\right]
$$

which is represented as the Feynman diagram shown in Fig. 2. In this expression, we use the relation $\gamma_{45} G(K)^{\mathrm{T}} \gamma_{45}=G(K)$.

Meanwhile, we can parametrize the terms in the free energy accordingly:

$$
F_{2}\left(\Delta_{a}\right)=r_{a}\left|\Delta_{a}\right|^{2}
$$

Choosing the specific configurations,

$$
\begin{aligned}
\Delta_{e_{g}}^{1} & =(1,0), \Delta_{e_{g}}^{2}=(0,1), \Delta_{t_{2 g}}^{1} \\
& =(1,0,0), \Delta_{t_{2 g}}^{2}=(0,1,0), \Delta_{t_{2 g}}^{3}=(0,0,1),
\end{aligned}
$$

we apply Eq. (A3) and

$$
\begin{aligned}
& F_{2}\left(\Delta_{e_{g}}^{1}\right)=r_{1}, F_{2}\left(\Delta_{e_{g}}^{2}\right)=r_{2}, \\
& F_{2}\left(\Delta_{t_{2 g}}^{1}\right)=r_{3}, F_{2}\left(\Delta_{t_{2 g}}^{2}\right)=r_{4}, F_{2}\left(\Delta_{t_{2 g}}^{3}\right)=r_{5}
\end{aligned}
$$

to get the coefficients $r_{a}$. Then we can write the coefficients as follows with $\hat{k}_{0}=k_{0} / T=2 \pi(n+1 / 2), \hat{c}_{i}=c_{i} / T$, and $\hat{\mu}=\mu / T$ :

$$
r_{a}=\frac{1}{g}+T^{1 / 2} \int_{\boldsymbol{k}} \sum_{n} \frac{2\left\{\sum_{i}\left[\hat{c}_{i} d_{i}(\boldsymbol{k})\right]^{2}-2\left[\hat{c}_{a} d_{a}(\boldsymbol{k})\right]^{2}-\left(\hat{c}_{0} k^{2}-\hat{\mu}\right)^{2}-\hat{k}_{0}^{2}\right\}}{\left\{\sum_{i}\left[\hat{c}_{i} d_{i}(\boldsymbol{k})\right]^{2}-\left(\hat{c}_{0} k^{2}-\hat{\mu}+i \hat{k}_{0}\right)^{2}\right\}\left\{\sum_{i}\left[\hat{c}_{i} d_{i}(\boldsymbol{k})\right]^{2}-\left(\hat{c}_{0} k^{2}-\hat{\mu}-i \hat{k}_{0}\right)^{2}\right\}} .
$$

Remarkably, $r_{a}-r_{b}$ can be simply expressed as follows:

$$
r_{a}-r_{b}=T^{1 / 2} \int_{\boldsymbol{k}} \sum_{n} \frac{-4\left[\hat{c}_{a} d_{a}(\boldsymbol{k})\right]^{2}+4\left[\hat{c}_{b} d_{b}(\boldsymbol{k})\right]^{2}}{\left\{\sum_{i}\left[\hat{c}_{i} d_{i}(\boldsymbol{k})\right]^{2}-\left(\hat{c}_{0} k^{2}-\hat{\mu}+i \hat{k}_{0}\right)^{2}\right\}\left\{\sum_{i}\left[\hat{c}_{i} d_{i}(\boldsymbol{k})\right]^{2}-\left(\hat{c}_{0} k^{2}-\hat{\mu}-i \hat{k}_{0}\right)^{2}\right\}} .
$$

In Fig. 3, we plot the evolution of coefficients $\tilde{r}_{a} \equiv r_{a} / T^{1 / 2}-1 / g$ as a function of $\hat{O}_{20} \equiv \hat{c}_{5}-\hat{c}_{\eta}$, with $\hat{c}_{0}=-2000(a / \pi)^{2}, \hat{c}_{e_{g}} \equiv \hat{c}_{1}=\hat{c}_{2}=-2000 / 3(a / \pi)^{2}, \hat{c}_{\eta} \equiv$ $\hat{c}_{3}=\hat{c}_{4}=\left(-1000 / 3-\hat{O}_{20}\right)(a / \pi)^{2}, \quad \hat{c}_{5}=-1000 / 3(a / \pi)^{2}$, and $\hat{\mu}=-200$ as appropriate for PrBi. First, Fig. 3 clearly shows that the instability towards $\Delta_{e_{g}}$ pairing is stronger than $\Delta_{t_{2 g}}$ pairing, i.e., $\tilde{r}_{e_{g}} \equiv \tilde{r}_{1}=\tilde{r}_{2}<\tilde{r}_{t_{2 g}} \equiv \tilde{r}_{3}=\tilde{r}_{4}=\tilde{r}_{5}$, with $\hat{O}_{20}=0$ for cubic symmetry as stated in the main text. Moreover, Fig. 3 also shows that the instability towards $\Delta_{2}$ pairing becomes stronger than $\Delta_{1}$ pairing, i.e., $\tilde{r}_{2}<\tilde{r}_{1}$, as soon as $\mathrm{O}_{20}$-type ferroquadrupolar order becomes finite, $\hat{O}_{20} \neq 0$. Finally, it tells us that the instability towards $\Delta_{\eta}$ pairing becomes stronger than $\Delta_{2}$ pairing, $\tilde{r}_{\eta} \equiv \tilde{r}_{3}=\tilde{r}_{4}<\tilde{r}_{2}$, for $\hat{O}_{20}>\hat{c}_{5}-\hat{c}_{e_{g}}=1000 / 3(a / \pi)^{2}$.

\section{Quartic coefficients}

In this section, we compute the coefficient of the quartic terms $q_{a}$ in the Ginzburg-Landau free energy $F_{\eta}$. Let $F_{4, \eta}\left(\Delta_{\eta}\right)$ be the contribution to the free energy which contains the fourth power of $\Delta_{3}$ and $\Delta_{4}$. We have

$$
F_{4, \eta}\left(\Delta_{\eta}\right)=\frac{1}{4} \sum_{a, b, c, d} L_{a, b, c, d} \Delta_{a} \Delta_{b}^{*} \Delta_{c} \Delta_{d}^{*}
$$

with

$$
L_{a, b, c, d}=T \sum_{k_{0}} \int_{k}^{\Lambda} \operatorname{tr}\left[G(K) \gamma_{a} G(-K) \gamma_{c} G(K) \gamma_{a} G(-K) \gamma_{d}\right]
$$




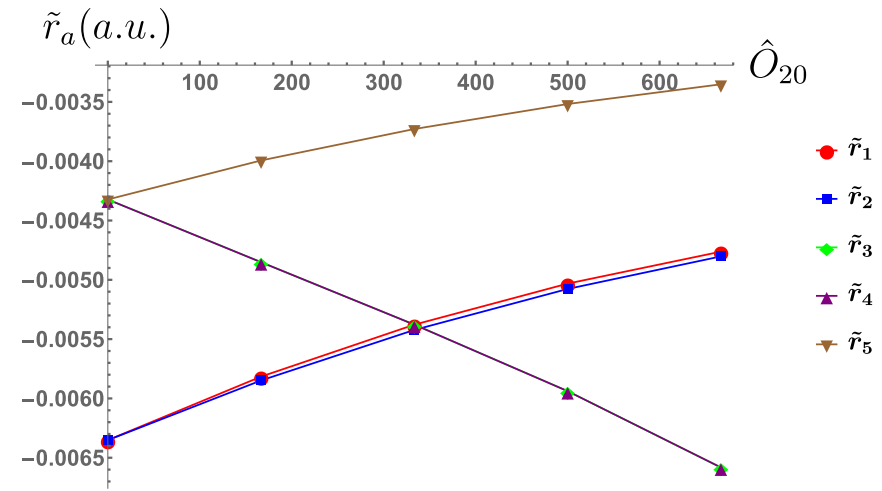

FIG. 3. Plot of coefficients $\tilde{r}_{a} \equiv r_{a} / T^{1 / 2}-1 / g$ as a function of $\hat{O}_{20}\left[(a / \pi)^{2}\right] \quad$ with $\quad \hat{c}_{0}=-2000(a / \pi)^{2}, \quad \hat{c}_{e_{g}} \equiv \hat{c}_{1}=\hat{c}_{2}=$ $-2000 / 3(a / \pi)^{2}, \quad \hat{c}_{\eta} \equiv \hat{c}_{3}=\hat{c}_{4}=\left(-1000 / 3-\hat{O}_{20}\right)(a / \pi)^{2}$, $\hat{c}_{5}=-1000 / 3(a / \pi)^{2}$, and $\hat{\mu}=-200$. These numbers are relevant to $\operatorname{PrBi}[60,61]$.
On the other hand, we can parametrize $F_{4, \eta}\left(\Delta_{\eta}\right)$ accordingly:

$$
F_{4, \eta}\left(\Delta_{\eta}\right)=q_{1}\left(\Delta_{\eta} \Delta_{\eta}^{*}\right)^{2}+q_{2}\left|\Delta_{\eta} \Delta_{\eta}\right|^{2}+q_{3}\left(\left|\Delta_{3}\right|^{4}+\left|\Delta_{4}\right|^{4}\right) .
$$

Choosing the specific configurations

$$
\Delta_{\eta}^{1}=(1,0), \Delta_{\eta}^{2}=\frac{1}{\sqrt{2}}(1,1), \Delta_{\eta}^{3}=\frac{1}{\sqrt{2}}(1, i),
$$

we apply Eq. (A9) and

$$
\begin{aligned}
F_{4, \eta}\left(\Delta_{\eta}^{1}\right) & =q_{1}+q_{2}+q_{3}, F_{4, \eta}\left(\Delta_{\eta}^{2}\right) \\
& =q_{1}+q_{2}+\frac{q_{3}}{2}, F_{4, \eta}\left(\Delta_{\eta}^{3}\right)=q_{1}+\frac{q_{3}}{2}
\end{aligned}
$$

to get the coefficients $q_{a}$. By introducing $\hat{D}_{i} \equiv \hat{c}_{i} d_{i}(\boldsymbol{k})$, we can write the coefficients as follows when $\hat{c}_{0}=0$ and $\hat{\mu}=0$ :

$$
q_{a}=\frac{1}{T^{3 / 2}} \int_{k} \sum_{n} \frac{\hat{p}_{a}}{\left[\sum_{i} \hat{D}_{i}^{2}+\hat{k}_{0}^{2}\right]^{2}},
$$

where

$$
\begin{aligned}
\hat{p}_{1}= & 2\left[2\left(-\hat{D}_{3}^{2}+2 \hat{D}_{4} \hat{D}_{3}+\hat{D}_{4}^{2}-3 \hat{D}_{5}^{2}\right) \hat{k}_{0}^{2}+\hat{D}_{1}^{4}+\hat{D}_{2}^{4}-\hat{D}_{3}^{4}-\hat{D}_{4}^{4}+\hat{D}_{5}^{4}+6 \hat{D}_{3}^{2} \hat{D}_{4}^{2}+2 \hat{D}_{3}^{2} \hat{D}_{5}^{2}-2 \hat{D}_{4}^{2} \hat{D}_{5}^{2}-4 \hat{D}_{3} \hat{D}_{4} \hat{D}_{5}^{2}\right. \\
& \left.+2 \hat{D}_{2}^{2}\left(\hat{D}_{3}^{2}-2 \hat{D}_{4} \hat{D}_{3}-\hat{D}_{4}^{2}+\hat{D}_{5}^{2}-3 \hat{k}_{0}^{2}\right)+2 \hat{D}_{1}^{2}\left(\hat{D}_{2}^{2}+\hat{D}_{3}^{2}-\hat{D}_{4}^{2}+\hat{D}_{5}^{2}-2 \hat{D}_{3} \hat{D}_{4}-3 \hat{k}_{0}^{2}\right)+\hat{k}_{0}^{4}\right], \\
\hat{p}_{2}= & -2\left(\hat{D}_{3}^{2}-4 \hat{D}_{4} \hat{D}_{3}+\hat{D}_{4}^{2}-3 \hat{D}_{5}^{2}\right) \hat{k}_{0}^{2}-\hat{D}_{1}^{4}-\hat{D}_{2}^{4}-\hat{D}_{3}^{4}-\hat{D}_{4}^{4}-\hat{D}_{5}^{4}+6 \hat{D}_{3}^{2} \hat{D}_{4}^{2}-2 \hat{D}_{3}^{2} \hat{D}_{5}^{2}-2 \hat{D}_{4}^{2} \hat{D}_{5}^{2}-8 \hat{D}_{3} \hat{D}_{4} \hat{D}_{5}^{2} \\
& -2 \hat{D}_{2}^{2}\left(\hat{D}_{3}^{2}+4 \hat{D}_{4} \hat{D}_{3}+\hat{D}_{4}^{2}+\hat{D}_{5}^{2}-3 \hat{k}_{0}^{2}\right)-2 \hat{D}_{1}^{2}\left(\hat{D}_{2}^{2}+\hat{D}_{3}^{2}+\hat{D}_{4}^{2}+\hat{D}_{5}^{2}+4 \hat{D}_{3} \hat{D}_{4}-3 \hat{k}_{0}^{2}\right)-\hat{k}_{0}^{4}, \\
\hat{p}_{3}= & 4\left(\hat{D}_{3}^{2}-2 \hat{D}_{4} \hat{D}_{3}-\hat{D}_{4}^{2}\right)\left(-2 \hat{D}_{1}^{2}-2 \hat{D}_{2}^{2}+\hat{D}_{3}^{2}-\hat{D}_{4}^{2}-2 \hat{D}_{5}^{2}+2 \hat{D}_{3} \hat{D}_{4}+2 \hat{k}_{0}^{2}\right) .
\end{aligned}
$$

Using the above equations, we plot the coefficients as a function of $\hat{O}_{20}$ with $\hat{c}_{e_{g}}=-2000 / 3(a / \pi)^{2}, \hat{c}_{\eta}=$ $\left(-1000 / 3-\hat{O}_{20}\right)(a / \pi)^{2}$, and $\hat{c}_{5}=-1000 / 3(a / \pi)^{2}$ in Fig. 4. First, the result indicates that the free energy is stable $\left(q_{1} T^{3 / 2}>0\right)$. Moreover, the negative sign of $q_{2}$ and $q_{3}$ clearly shows that the system stabilizes $d_{y z}$ pairing when $\hat{c}_{0}=0$ and $\hat{\mu}=0$.

$\tilde{q}_{a}(a . u$.

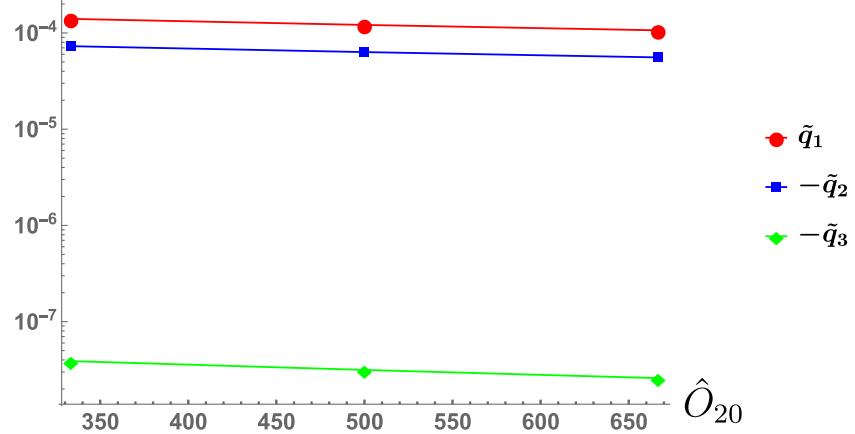

FIG. 4. Plot of coefficients $\tilde{q}_{i} \equiv q_{i} T^{3 / 2}$ as a function of $\hat{O}_{20}\left[(a / \pi)^{2}\right] \quad$ with $\quad \hat{c}_{e_{g}}=-2000 / 3(a / \pi)^{2}, \quad \hat{c}_{\eta}=(-1000 / 3-$ $\left.\hat{O}_{20}\right)(a / \pi)^{2}$, and $\hat{c}_{5}=-1000 / 3(a / \pi)^{2}$.

\section{Ginzburg-Landau free energy near the phase boundary}

In this section, we derive the Ginzburg-Landau free energy near the phase boundary where the instabilities towards the $\Delta_{2}$ and $\Delta_{\eta}$ pairing are comparable (i.e., $r_{2} \sim r_{\eta}$ ) and analyze it to describe the phase transition. Near the phase boundary, the free energy in point group $D_{4 h}$ is given by

$$
\begin{aligned}
F_{D_{4 h}}= & r_{2}\left|\Delta_{2}\right|^{2}+r_{\eta} \Delta_{\eta} \Delta_{\eta}^{*}+p\left|\Delta_{2}\right|^{4}+q_{1}\left(\Delta_{\eta} \Delta_{\eta}^{*}\right)^{2} \\
& +q_{2}\left|\Delta_{\eta} \Delta_{\eta}\right|^{2}+q_{3}\left(\left|\Delta_{3}\right|^{4}+\left|\Delta_{4}\right|^{4}\right) \\
& +m_{1}\left[\left(\Delta_{\eta} \Delta_{\eta}\right)\left(\Delta_{2}^{*}\right)^{2}+\text { c.c. }\right]+m_{2}\left(\Delta_{\eta} \Delta_{\eta}^{*}\right)\left|\Delta_{2}\right|^{2} .
\end{aligned}
$$

Depending on the coefficients, the free energy of Eq. (A12) can stabilize five mixed states, which can be represented accordingly:

$$
\begin{aligned}
& \Delta_{D_{4 h}}^{1}=\left(\Delta_{2}, \eta, 0\right), \Delta_{D_{4 h}}^{2}=\left(i \Delta_{2}, \eta, 0\right), \\
& \Delta_{D_{4 h}}^{3}=\left(\Delta_{2}, \frac{\eta}{\sqrt{2}}, \frac{\eta}{\sqrt{2}}\right), \Delta_{D_{4 h}}^{4}=\left(i \Delta_{2}, \frac{\eta}{\sqrt{2}}, \frac{\eta}{\sqrt{2}}\right), \\
& \Delta_{D_{4 h}}^{5}=\left(\Delta_{2}, \frac{\eta}{\sqrt{2}}, i \frac{\eta}{\sqrt{2}}\right),
\end{aligned}
$$

where $\Delta_{D_{4 h}} \equiv\left(\Delta_{2}, \Delta_{3}, \Delta_{4}\right)$ and $\Delta_{2}$ and $\eta$ are assumed to be real numbers without loss of generality. After inserting Eq. (A13) into Eq. (A12), one gets the simplified free energy, 


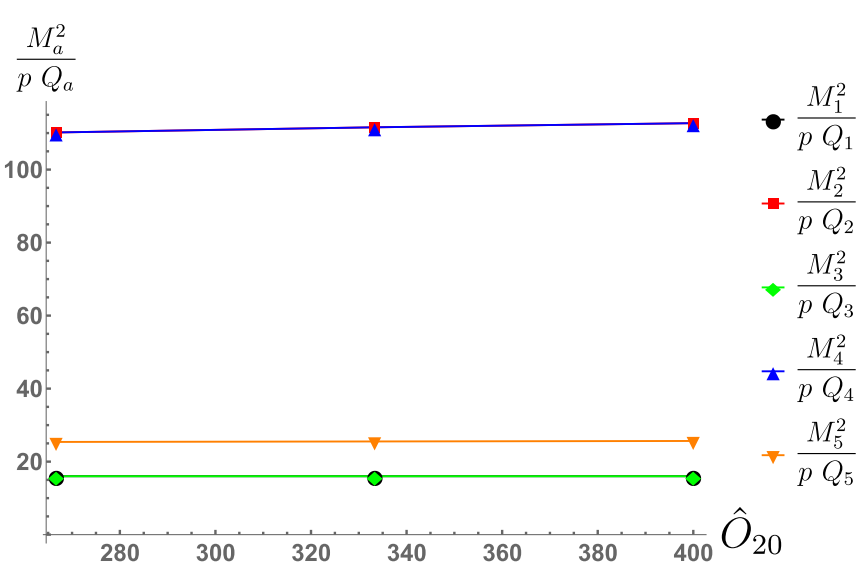

FIG. 5. Evolution of $M_{a}^{2} / p Q_{a}$ as a function of $\hat{O}_{20}\left[(a / \pi)^{2}\right]$ with $\hat{c}_{0}=0, \hat{c}_{e_{g}}=-2000 / 3(a / \pi)^{2}, \hat{c}_{\eta}=\left(-1000 / 3-\hat{O}_{20}\right)(a / \pi)^{2}, \hat{c}_{5}=$ $-1000 / 3(a / \pi)^{2}$, and $\hat{\mu}=0$.

which is given by

$$
\begin{aligned}
F_{D_{4 h}}= & r_{2} \Delta_{2}^{2}+r_{\eta} \eta^{2}+p \Delta_{2}^{4}+Q^{a}\left(q_{1}, q_{2}, q_{3}\right) \eta^{4} \\
& +M^{a}\left(m_{1}, m_{2}\right) \Delta^{2} \eta^{2},
\end{aligned}
$$

with

$$
\begin{aligned}
& \left(Q^{1}, M^{1}\right)=\left(q_{1}+q_{2}+q_{3}, 2 m_{1}+m_{2}\right), \\
& \left(Q^{2}, M^{2}\right)=\left(q_{1}+q_{2}+q_{3},-2 m_{1}+m_{2}\right), \\
& \left(Q^{3}, M^{3}\right)=\left(q_{1}+q_{2}+\frac{q_{3}}{2}, 2 m_{1}+m_{2}\right), \\
& \left(Q^{4}, M^{4}\right)=\left(q_{1}+q_{2}+\frac{q_{3}}{2},-2 m_{1}+m_{2}\right), \\
& \left(Q^{5}, M^{5}\right)=\left(q_{1}+\frac{q_{3}}{2}, m_{2}\right) .
\end{aligned}
$$

If $\left[M_{a}\left(m_{1}, m_{2}\right)\right]^{2}>p Q_{a}\left(q_{1}, q_{2}, q_{3}\right)$, the phase competition between the $\Delta_{2}$ and $\Delta_{\eta}$ pairing states is sufficiently strong that both states are separated by a first-order transition [75,76], and therefore, the free energy of Eq. (A14) does not stabilize the mixed state $\Delta_{D_{4}}^{a}$.

Using Eq. (A8) and the free energy of Eq. (A12), one can compute the quartic coefficients, $p, q_{1}, q_{2}, q_{3}, m_{1}$, and $m_{2}$, by following the procedure given in the previous section. Figure 5 shows the evolution of $M_{a}^{2} / p Q_{a}$ as a function of $\hat{O}_{20}$ with $\hat{c}_{0}=0, \hat{c}_{e_{g}}=-2000 / 3(a / \pi)^{2}, \hat{c}_{\eta}=(-1000 / 3-$ $\left.\hat{O}_{20}\right)(a / \pi)^{2}, \quad \hat{c}_{5}=-1000 / 3(a / \pi)^{2}$, and $\hat{\mu}=0$. The plot shows that $\left[M_{a}\left(m_{1}, m_{2}\right)\right]^{2}>p Q_{a}\left(q_{1}, q_{2}, q_{3}\right)$ and the free energy of Eq. (A12) is minimized such that the pairing channel with the smaller quadratic coefficient, $r_{2}$ or $r_{\eta}$, completely suppresses the other, and the phase transition is of first order.

\section{APPENDIX B: CALCULATION OF MEAN-FIELD ENERGY AT ZERO TEMPERATURE}

Here, we compare the energy of possible states by solving the self-consistent gap equations numerically. By doing that, we achieve the zero-temperature mean-field phase diagram, which is given in the main text.

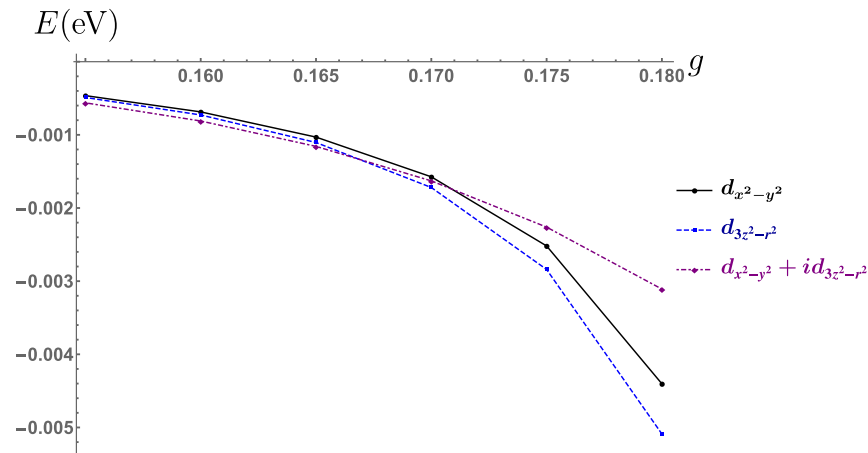

FIG. 6. Evolution of mean-field energy $E$ of $d_{x^{2}-y^{2}}$ (solid black line), $d_{3 z^{2}-r^{2}}$ (dashed blue line), and $d_{x^{2}-y^{2}}+i d_{3 z^{2}-r^{2}}$ (dot-dashed purple line) states as a function of the interaction strength $g\left(a^{3} \mathrm{eV}\right)$ at zero temperature. Other specific parameters, which are used to obtain the plot, are $c_{0}=-6(a / \pi)^{2} \mathrm{eV}, c_{e_{g}}=-2(a / \pi)^{2} \mathrm{eV}, c_{t_{2 g}}=$ $-1(a / \pi)^{2} \mathrm{eV}$, and $\mu=-0.6 \mathrm{eV}$. These numbers are relevant to PrBi $[60,61]$.

\section{Cubic-symmetric Luttinger model without quadrupolar order}

In Appendix A, we found that the instability towards the $\Delta_{e_{g}}$ pairing is stronger than the $\Delta_{t_{2 g}}$ pairing when $\left|c_{e_{g}}\right|>\left|c_{t_{2 g}}\right|$. For the $\Delta_{e_{g}}$ pairing, there are three possible superconducting states for the cubic-symmetric case: $\Delta_{e_{s}}=(1,0),(0,1)$, and $(1, i)$. Having classified the possible states, we compute their mean-field energy. We start with mean-field BCS Hamiltonian $H_{\mathrm{BCS}} . H_{\mathrm{BCS}}$ is written as

$$
\begin{aligned}
H_{\mathrm{BCS}}= & \sum_{\boldsymbol{k}}\left\{\psi_{\boldsymbol{k}}^{\dagger}\left[c_{0} k^{2}+\sum_{i=1}^{5} c_{i} d_{i}(\boldsymbol{k}) \gamma_{i}-\mu\right] \psi_{\boldsymbol{k}}\right. \\
& \left.-\sum_{i=1}^{5}\left(\Delta_{i} \psi_{\boldsymbol{k}}^{\dagger} \gamma_{45} \gamma_{i} \psi_{-\boldsymbol{k}}^{*}+\Delta_{i}^{*} \psi_{-\boldsymbol{k}}^{T} \gamma_{45} \gamma_{i} \psi_{\boldsymbol{k}}\right)\right\} \\
& +\frac{1}{g} \Delta_{i} \Delta_{i}^{*},
\end{aligned}
$$

and the self-consistent gap equations are written as

$$
\Delta_{i}=g\left(\psi_{-k}^{T} \gamma_{45} \gamma_{a} \psi_{\boldsymbol{k}}\right\rangle .
$$

Then, we can compute the energy $E$ of the three states by taking the expectation value of $H_{\mathrm{BCS}}$ with $\Delta_{i}$, which are numerically obtained using Eq. (B2) at a given temperature. Figure 6 shows the evolution of mean-field energy $E$ for the three states as a function of the interaction strength $g$ at zero temperature. The plot clearly shows that a phase transition occurs from the $d_{x^{2}-y^{2}}+i d_{3 z^{2}-r^{2}}$ state to the $d_{3 z^{2}-r^{2}}$ state at $g \sim 0.167 a^{3} \mathrm{eV}$.

\section{Luttinger model with quadrupolar order}

In Appendix A, we found that the instability towards the $\Delta_{\eta}$ pairing is stronger than the other pairings when $\left|c_{\eta}\right|>$ $\left|c_{e_{g}}\right|>\left|c_{5}\right|$. For the $\Delta_{\eta}$ pairing, there are three possible superconducting states: $\Delta_{\eta}=(1,0),(1,1)$, and $(1, i)$. In Fig. 7 , we plot the evolution of $E$ for the three states at zero temperature using Eqs. (B1) and (B2). 


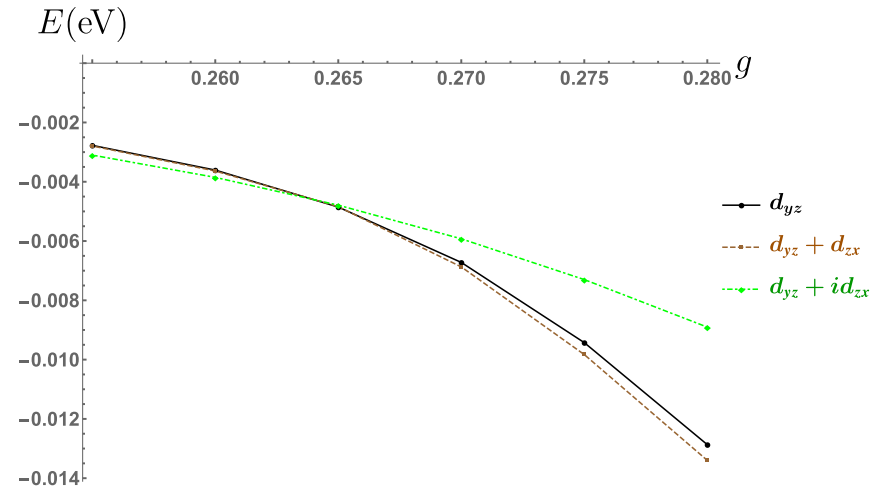

FIG. 7. Evolution of mean-field energy $E$ of $d_{y z}$ (solid black line), $d_{y z}+d_{z x}$ (dashed brown line), and $d_{y z}+i d_{z x}$ (dot-dashed green line) states as a function of the interaction strength $g\left(a^{3} \mathrm{eV}\right)$ at zero temperature. Other specific parameters used to attain this plot are $c_{0}=-6(a / \pi)^{2} \mathrm{eV}, c_{e_{g}}=-2(a / \pi)^{2} \mathrm{eV}, c_{\eta}=-3(a / \pi)^{2} \mathrm{eV}, c_{5}=$ $-1(a / \pi)^{2} \mathrm{eV}$, and $\mu=-0.6 \mathrm{eV}$.

\section{APPENDIX C: KONDO COUPLING AND FERMI-SURFACE DISTORTION}

In this section, we derive the effective Kondo coupling between the quadrupolar order parameters and the itinerant $j=3 / 2$ electrons for the interpenetrating fcc lattice system. We start by introducing the Kondo model where the quadrupolar order parameters $O_{22}$ and $O_{20}$ and the itinerant $t_{2 g}$ electrons couple as follows:

$$
H_{K}=J_{K} \sum_{\langle i, j\rangle} \sum_{a, \alpha}\left(O_{22} \Gamma_{1, i j}^{a} c_{i a \alpha}^{\dagger} c_{j a \alpha}+O_{20} \Gamma_{2, i j}^{a} c_{i a \alpha}^{\dagger} c_{j a \alpha}\right) .
$$

Here, $c_{i a \alpha}^{\dagger}$ and $c_{i a \alpha}$ are the electron creation and annihilation operators at site $i$ with orbital $a \in(x, y, z)$ and $\operatorname{spin} \alpha \in(\uparrow, \downarrow)$. We consider the case where the quadrupolar degrees of freedom from the localized electron reside in one fcc lattice and the itinerant electrons with $p$ orbitals reside in another fcc lattice as in Fig. 8. Then one of the Kondo coupling terms, which couples itinerant electrons in the $p_{x}$ orbital with

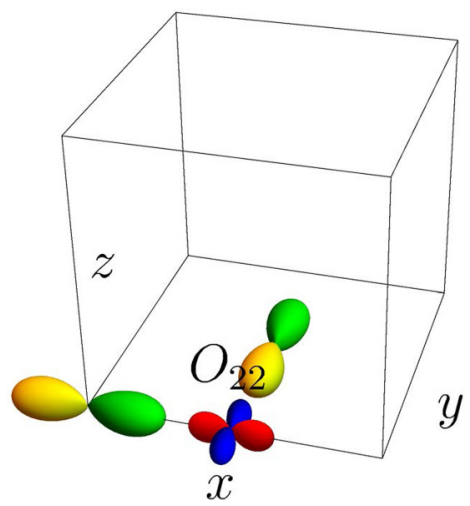

FIG. 8. Position of the quadrupolar degrees of freedom and the itinerant electrons with $p$ orbitals in the interpenetrating fcc lattice system. One of two quadrupole moments, $O_{22}$, is colored red and blue. The $p_{x}$ orbital is colored green and yellow. other itinerant electrons in the $p_{y}$ orbital residing on nearestneighbor sites, can be written as

$$
\begin{aligned}
H_{i x y}= & J_{K} \sum_{\alpha} O_{22}\left(c_{i_{x} y \alpha}^{\dagger} c_{i x \alpha}-c_{i_{y} x \alpha}^{\dagger} c_{i y \alpha}+c_{i_{-x} y \alpha}^{\dagger} c_{i x \alpha}\right. \\
& \left.-c_{i_{-y} x \alpha}^{\dagger} c_{i y \alpha}\right),
\end{aligned}
$$

where the site index $i_{a}$ represents the nearest neighbor of site $i$ in the $a$ direction. Here, the minus sign for the second term comes from $\mathrm{O}_{22}$, which transforms as $\mathrm{O}_{22} \rightarrow-\mathrm{O}_{22}$ under $C_{4 z}$ ( $\pi / 2$ rotation about the $z$ axis). Using $C_{31}$ rotation $[2 \pi / 3$ rotation along the (111) direction], we can write symmetryrelated terms as

$$
\begin{aligned}
H_{i y z}= & J_{K} \sum_{\alpha}\left(-\frac{1}{2} O_{22}-\frac{\sqrt{3}}{2} O_{20}\right)\left(c_{i_{y} z \alpha}^{\dagger} c_{i y \alpha}-c_{i_{z} y \alpha}^{\dagger} c_{i z \alpha}\right. \\
& \left.+c_{i_{-y} z \alpha}^{\dagger} c_{i y \alpha}-c_{i_{-z} y \alpha}^{\dagger} c_{i z \alpha}\right), \\
H_{i z x}= & J_{K} \sum_{\alpha}\left(-\frac{1}{2} O_{22}+\frac{\sqrt{3}}{2} O_{20}\right)\left(c_{i_{z} x \alpha}^{\dagger} c_{i z \alpha}-c_{i_{x} z \alpha}^{\dagger} c_{i x \alpha}\right. \\
& \left.+c_{i_{-z} x \alpha}^{\dagger} c_{i z \alpha}-c_{i_{-x} z \alpha}^{\dagger} c_{i x \alpha}\right) .
\end{aligned}
$$

After Fourier transforming the Hamiltonian, $H_{K}=\sum_{i}\left(H_{i x y}+\right.$ $\left.H_{i y z}+H_{i z x}\right)$, and expanding around $\boldsymbol{k}=0$, the Kondo Hamiltonian is written as

$$
\begin{aligned}
H_{K}(\boldsymbol{k})= & J_{K}\left(\frac{a}{\pi}\right)^{2} \sum_{\boldsymbol{k}} \sum_{\alpha}\left[O _ { 2 2 } ( - k _ { x } ^ { 2 } - k _ { y } ^ { 2 } - k _ { x } k _ { y } ) \left(c_{\boldsymbol{k} x \alpha}^{\dagger} c_{\boldsymbol{k} y \alpha}\right.\right. \\
& \left.+c_{\boldsymbol{k} y \alpha}^{\dagger} c_{\boldsymbol{k} x \alpha}\right)+\left(-\frac{1}{2} O_{22}-\frac{\sqrt{3}}{2} O_{20}\right) \\
& \times\left(-k_{y}^{2}-k_{z}^{2}-k_{y} k_{z}\right)\left(c_{\boldsymbol{k} y \alpha}^{\dagger} c_{\boldsymbol{k} z \alpha}+c_{\boldsymbol{k} z \alpha}^{\dagger} c_{\boldsymbol{k} y \alpha}\right) \\
& +\left(-\frac{1}{2} O_{22}+\frac{\sqrt{3}}{2} O_{20}\right)\left(-k_{z}^{2}-k_{x}^{2}-k_{z} k_{x}\right)\left(c_{\boldsymbol{k} z \alpha}^{\dagger} c_{\boldsymbol{k} x \alpha}\right. \\
& \left.\left.+c_{\boldsymbol{k} x \alpha}^{\dagger} c_{\boldsymbol{k} z \alpha}\right)\right] .
\end{aligned}
$$

By projecting onto the $j=3 / 2$ basis with the projection operator $P_{j=3 / 2}$, one gets the following Kondo coupling:

$$
\begin{aligned}
\tilde{H}_{K}(\boldsymbol{k})= & P_{j=3 / 2} H_{K}(\boldsymbol{k}) P_{j=3 / 2} \\
= & \tilde{J}_{K}\left[\left(\sqrt{3} O_{20}+O_{22}\right) d_{3}(\boldsymbol{k}) \gamma_{3}\right. \\
& \left.+\left(\sqrt{3} O_{20}-O_{22}\right) d_{4}(\boldsymbol{k}) \gamma_{4}-2 O_{22} d_{5}(\boldsymbol{k}) \gamma_{5}\right]
\end{aligned}
$$

in the four-component spinor basis $\psi$. 
[1] X.-L. Qi, T. L. Hughes, S. Raghu, and S.-C. Zhang, Phys. Rev. Lett. 102, 187001 (2009).

[2] X.-L. Qi, T. L. Hughes, and S.-C. Zhang, Phys. Rev. B 81, 134508 (2010).

[3] M. Z. Hasan and C. L. Kane, Rev. Mod. Phys. 82, 3045 (2010).

[4] M. Sato and Y. Ando, Rep. Prog. Phys. 80, 076501 (2017).

[5] L. Fu and C. L. Kane, Phys. Rev. Lett. 100, 096407 (2008).

[6] S. B. Chung, X.-L. Qi, J. Maciejko, and S.-C. Zhang, Phys. Rev. B 83, 100512(R) (2011).

[7] G. Y. Cho, J. H. Bardarson, Y.-M. Lu, and J. E. Moore, Phys. Rev. B 86, 214514 (2012).

[8] G. Bednik, A. A. Zyuzin, and A. A. Burkov, Phys. Rev. B 92, 035153 (2015).

[9] Y. Li and F. D. M. Haldane, Phys. Rev. Lett. 120, 067003 (2018).

[10] S. Nadj-Perge, I. K. Drozdov, J. Li, H. Chen, S. Jeon, J. Seo, A. H. MacDonald, B. A. Bernevig, and A. Yazdani, Science 346, 602 (2014).

[11] Q. L. He, L. Pan, A. L. Stern, E. C. Burks, X. Che, G. Yin, J. Wang, B. Lian, Q. Zhou, E. S. Choi et al., Science 357, 294 (2017).

[12] P. M. R. Brydon, L. Wang, M. Weinert, and D. F. Agterberg, Phys. Rev. Lett. 116, 177001 (2016).

[13] D. F. Agterberg, P. M. R. Brydon, and C. Timm, Phys. Rev. Lett. 118, 127001 (2017).

[14] C. Timm, A. P. Schnyder, D. F. Agterberg, and P. M. R. Brydon, Phys. Rev. B 96, 094526 (2017).

[15] P. M. R. Brydon, D. F. Agterberg, H. Menke, and C. Timm, Phys. Rev. B 98, 224509 (2018).

[16] H. Menke, C. Timm, and P. M. R. Brydon, Phys. Rev. B 100, 224505 (2019).

[17] B. Roy, S. A. A. Ghorashi, M. S. Foster, and A. H. Nevidomskyy, Phys. Rev. B 99, 054505 (2019).

[18] A. Szabo, R. Moessner, and B. Roy, arXiv:1811.12415.

[19] I. Boettcher and I. F. Herbut, Phys. Rev. Lett. 120, 057002 (2018).

[20] I. F. Herbut, I. Boettcher, and S. Mandal, Phys. Rev. B 100, 104503 (2019).

[21] G. B. Sim, A. Mishra, M. J. Park, Y. B. Kim, G. Y. Cho, and S. B. Lee, Phys. Rev. B 100, 064509 (2019).

[22] J. W. F. Venderbos, L. Savary, J. Ruhman, P. A. Lee, and L. Fu, Phys. Rev. X 8, 011029 (2018).

[23] L. Savary, J. Ruhman, J. W. F. Venderbos, L. Fu, and P. A. Lee, Phys. Rev. B 96, 214514 (2017).

[24] W. Yang, Y. Li, and C. Wu, Phys. Rev. Lett. 117, 075301 (2016).

[25] C. Wu, J. Hu, and S.-C. Zhang, Int. J. Mod. Phys. B 24, 311 (2010).

[26] S. Tchoumakov, L. J. Godbout, and W. Witczak-Krempa, Phys. Rev. Research 2, 013230 (2020).

[27] M. A. Continentino, F. Deus, I. T. Padilha, and H. Caldas, Ann. Phys. (NY) 348, 1 (2014).

[28] G. A. Hamilton, M. J. Park, and M. J. Gilbert, Phys. Rev. B 100, 134512 (2019).

[29] M. Kriener, K. Segawa, Z. Ren, S. Sasaki, and Y. Ando, Phys. Rev. Lett. 106, 127004 (2011).

[30] S. Deng, L. Viola, and G. Ortiz, Phys. Rev. Lett. 108, 036803 (2012).

[31] T. Kawakami, T. Okamura, S. Kobayashi, and M. Sato, Phys. Rev. X 8, 041026 (2018).
[32] J. M. Link, I. Boettcher, and I. F. Herbut, Phys. Rev. B 101, 184503 (2020).

[33] Y.-P. Lin and R. M. Nandkishore, Phys. Rev. B 97, 134521 (2018).

[34] Y.-P. Lin, arXiv:1911.01436.

[35] G. Sim, M. J. Park, and S. Lee, arXiv:1909.04015.

[36] J. M. Luttinger and W. Kohn, Phys. Rev. 97, 869 (1955).

[37] J. Luttinger, Phys. Rev. 102, 1030 (1956).

[38] H. Oh and E.-G. Moon, arXiv:1911.08487.

[39] H. Kim, K. Wang, Y. Nakajima, R. Hu, S. Ziemak, P. Syers, L. Wang, H. Hodovanets, J. D. Denlinger, P. M. Brydon et al., Sci. Adv. 4, eaao4513 (2018).

[40] G. Goll, M. Marz, A. Hamann, T. Tomanic, K. Grube, T. Yoshino, and T. Takabatake, Phys. B (Amsterdam, Neth.) 403, 1065 (2008).

[41] Y. Nakajima, R. Hu, K. Kirshenbaum, A. Hughes, P. Syers, X. Wang, K. Wang, R. Wang, S. R. Saha, D. Pratt et al., Sci. Adv. 1, e1500242 (2015).

[42] M. Meinert, Phys. Rev. Lett. 116, 137001 (2016).

[43] T. Oguchi, Phys. Rev. B 63, 125115 (2001).

[44] H. Xiao, T. Hu, W. Liu, Y. L. Zhu, P. G. Li, G. Mu, J. Su, K. Li, and Z. Q. Mao, Phys. Rev. B 97, 224511 (2018).

[45] S. M. A. Radmanesh, C. Martin, Y. Zhu, X. Yin, H. Xiao, Z. Q. Mao, and L. Spinu, Phys. Rev. B 98, 241111(R) (2018).

[46] M. Hanawa, Y. Muraoka, T. Tayama, T. Sakakibara, J. Yamaura, and Z. Hiroi, Phys. Rev. Lett. 87, 187001 (2001).

[47] S.-W. Huang, H.-T. Jeng, J. Lin, W. Chang, J. Chen, G. Lee, H. Berger, H. Yang, and K. S. Liang, J. Phys.: Condens. Matter 21, 195602 (2009).

[48] J. Harter, Z. Zhao, J.-Q. Yan, D. Mandrus, and D. Hsieh, Science 356, 295 (2017).

[49] Y. Matsubayashi, T. Hasegawa, N. Ogita, J.-i. Yamaura, and Z. Hiroi, Phys. B (Amsterdam, Neth.) 536, 600 (2018).

[50] I. Ishii, H. Muneshige, S. Kamikawa, T. K. Fujita, T. Onimaru, N. Nagasawa, T. Takabatake, T. Suzuki, G. Ano, M. Akatsu et al., Phys. Rev. B 87, 205106 (2013).

[51] T. Onimaru, K. T. Matsumoto, Y. F. Inoue, K. Umeo, Y. Saiga, Y. Matsushita, R. Tamura, K. Nishimoto, I. Ishii, T. Suzuki et al., J. Phys. Soc. Jpn. 79, 033704 (2010).

[52] T. Onimaru, N. Nagasawa, K. T. Matsumoto, K. Wakiya, K. Umeo, S. Kittaka, T. Sakakibara, Y. Matsushita, and T. Takabatake, Phys. Rev. B 86, 184426 (2012).

[53] A. Sakai, K. Kuga, and S. Nakatsuji, J. Phys. Soc. Jpn. 81, 083702 (2012).

[54] T. Onimaru, K. T. Matsumoto, Y. F. Inoue, K. Umeo, T. Sakakibara, Y. Karaki, M. Kubota, and T. Takabatake, Phys. Rev. Lett. 106, 177001 (2011).

[55] M. Tsujimoto, Y. Matsumoto, T. Tomita, A. Sakai, and S. Nakatsuji, Phys. Rev. Lett. 113, 267001 (2014).

[56] T. J. Sato, S. Ibuka, Y. Nambu, T. Yamazaki, T. Hong, A. Sakai, and S. Nakatsuji, Phys. Rev. B 86, 184419 (2012).

[57] K. Matsubayashi, T. Tanaka, A. Sakai, S. Nakatsuji, Y. Kubo, and Y. Uwatoko, Phys. Rev. Lett. 109, 187004 (2012).

[58] X. He, C. Zhao, H. Yang, J. Wang, K. Cheng, S. Jiang, L. Zhao, Y. Li, C. Cao, Z. Zhu et al., Phys. Rev. B 101, 075106 (2020).

[59] I. Boettcher and I. F. Herbut, Phys. Rev. B 93, 205138 (2016).

[60] A. Vashist, R. K. Gopal, D. Srivastava, M. Karppinen, and Y. Singh, Phys. Rev. B 99, 245131 (2019).

[61] X. Duan, F. Wu, J. Chen, P. Zhang, Y. Liu, H. Yuan, and C. Cao, Commun. Phys. 1, 71 (2018). 
[62] M. Sigrist and K. Ueda, Rev. Mod. Phys. 63, 239 (1991).

[63] T. Bzdušek and M. Sigrist, Phys. Rev. B 96, 155105 (2017).

[64] K. Stevens, Proc. Phys. Soc. London, Sec. A 65, 209 (1952).

[65] K. Lea, M. Leask, and W. Wolf, J. Phys. Chem. Solids 23, 1381 (1962).

[66] G. L. Stamokostas and G. A. Fiete, Phys. Rev. B 97, 085150 (2018).

[67] S. B. Lee, S. Trebst, Y. B. Kim, and A. Paramekanti, Phys. Rev. B 98, 134447 (2018).

[68] F. Freyer, J. Attig, S. B. Lee, A. Paramekanti, S. Trebst, and Y. B. Kim, Phys. Rev. B 97, 115111 (2018).

[69] J. Ruan, S.-K. Jian, H. Yao, H. Zhang, S.-C. Zhang, and D. Xing, Nat. Commun. 7, 11136 (2016).
[70] D. Shao, J. Ruan, J. Wu, T. Chen, Z. Guo, H. Zhang, J. Sun, L. Sheng, and D. Xing, Phys. Rev. B 96, 075112 (2017).

[71] V. P. Mineev, K. Samokhin, and L. Landau, Introduction to Unconventional Superconductivity (CRC Press, Boca Raton, FL, 1999).

[72] C. Setty, S. Bhattacharyya, Y. Cao, A. Kreisel, and P. Hirschfeld, Nat. Commun. 11, 523 (2020).

[73] C. J. Lapp, G. Börner, and C. Timm, Phys. Rev. B 101, 024505 (2020).

[74] C. Setty, Y. Cao, A. Kreisel, S. Bhattacharyya, and P. Hirschfeld, arXiv:2004.00621.

[75] R. M. Fernandes and J. Schmalian, Phys. Rev. B 82, 014521 (2010).

[76] R. M. Fernandes and A. J. Millis, Phys. Rev. Lett. 111, 127001 (2013). 